\title{
A recurrent case of extraocular sebaceous carcinoma with distant organ metastasis
}

\author{
Byeong Soo Kwon, \\ Eui Han Chung, \\ Jin Woo Kim \\ Department of Plastic Surgery, Inje \\ University Busan Paik Hospital, Busan, \\ Korea
}

\begin{abstract}
Sebaceous carcinoma (SC) is a rare tumor, accounting for approximately $0.7 \%$ of skin cancers. SC can be classified as ocular SC (OSC) or extraocular SC (EOSC) depending on its location. Because EOSC accompanied by metastases is rare, there is a paucity of data about EOSC accompanied by metastasis. This study presents a case of an aggressive EOSC of the scalp with lymph node metastases. The patient underwent wide local excision of the primary tumor with a $1 \mathrm{~cm}$ safety margin and bilateral radical lymph node dissection. However, recurrence was observed 1 month after surgery. Radiation therapy and resection were performed for the recurrent tumor. However, distant metastases to both lungs eventually occurred. Here, we describe a rare recurrent case of EOSC of the scalp with distant organ metastasis with a review of the literature.
\end{abstract}

Keywords: Adenocarcinoma, sebaceous / Metastasis / Sebaceous gland neoplasms

\section{INTRODUCTION}

Sebaceous carcinoma (SC) is a rare tumor that grows in the adnexal epithelial cells of the sebaceous glands. It accounts for $0.7 \%$ of all skin cancers [1-3]. Risk factors for SC include advanced age, female sex, and Asian race [1]. SC can be classified as ocular SC (OSC) or extraocular SC (EOSC) depending on the location of its occurrence. SC commonly occurs in periocular regions with many sebaceous glands [2].

EOSCs are less common than OSCs, with EOSCs accompanied by metastases being even less common. It has been reported that only $1.4 \%$ of EOSCs are associated with regional lymph node metastases or distal organ metastases [4]. Considering that the most common sites of EOSC metastasis are regional lymph nodes [5], cases of EOSC with distant organ metastases are even rarer. For this reason, there is a paucity of data about EOSC accompanied by distant metastasis.

\section{Correspondence: Jin Woo Kim}

Department of Plastic Surgery, Inje University Busan Paik Hospital, 75 Bokji-ro,

Busanjin-gu, Busan 47392, Korea

E-mail: jinooda@hanmail.net

Received November 18, 2020 / Revised February 15, 2021 / Accepted February 15, 2021
We describe a rare recurrent case of EOSC. The patient was treated with wide local excision and radical lymph node dissection. However, distant metastases developed after 5 months. This study reports the clinical course of this rare case.

\section{CASE REPORT}

A 47-year-old man presenting with a painful mass on the left anterior scalp visited our hospital. The patient had a 40-year history of a yellow patch-like lesion on the same site with an irregular surface accompanied by itching and hair loss. The size of the lesion had not changed over time but had suddenly begun to grow 4 months before.

On physical examination, the patient had a hard, palpable, immobile mass measuring $7 \times 7 \mathrm{~cm}$ accompanied by bleeding, discharge, an irregular surface and a red ulcer on the left anterior scalp (Fig. 1). The patient also had bilateral palpable masses on the parotid gland and cervical lymph nodes. The patient had no specific underlying disease or family history of cancer. Due to the rapid growth of the mass, a malignant tumor was suspected. 


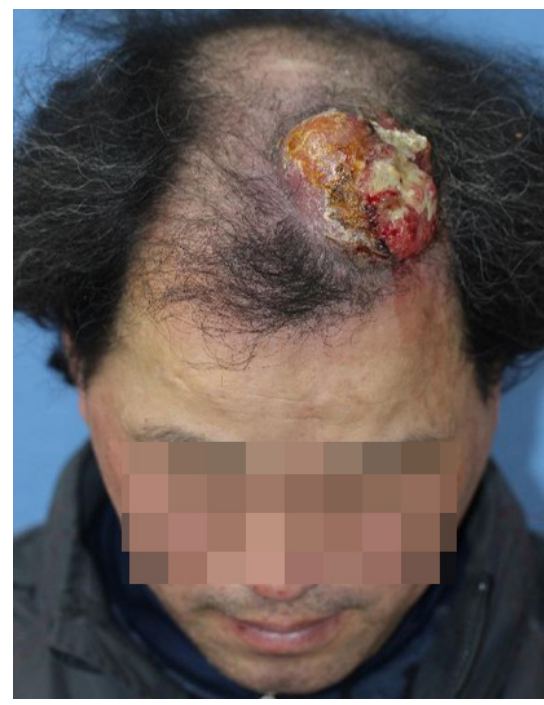

Fig. 1. Initial photograph. A 47-year-old man presented with a mass $7 \times 7 \mathrm{~cm}$ in size on the left anterior scalp.

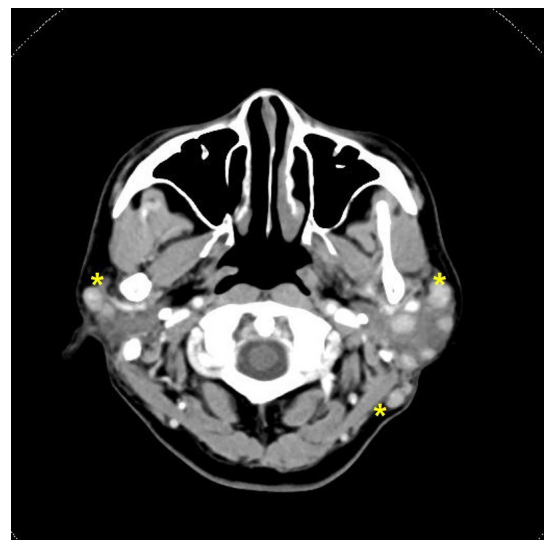

Fig. 2. Computed tomography (CT) scans of the lymph nodes. A CT image (yellow asterisks) shows several enhanced masses in the cervical lymph nodes and both parotid lymph nodes.

For further evaluation, a punch biopsy and computed tomography $(\mathrm{CT})$ were performed. On CT scans, the patient had an exophytic, heterogeneously enhancing mass measuring $6.9 \mathrm{~cm}$, accompanied by multiple masses on both parotid glands and the posterior neck (Fig. 2). On punch biopsy, there were signs that were suggestive of SC. The patient received a whole-body CT scan and a positron emission tomography scan for evaluation of distant metastases. However, no metastases were observed other than those to the parotid and cervical lymph nodes. The patient was therefore suspected of having SC of the scalp with lymph node metastases. The patient was planned for surgery but was lost to follow-up due to financial constraints.

At 3 months, the patient returned to us with bleeding from the scalp mass whose size had increased to $11 \times 11 \mathrm{~cm}$ (Fig. 3). The mass was accompanied by the presence of tissue necrosis,

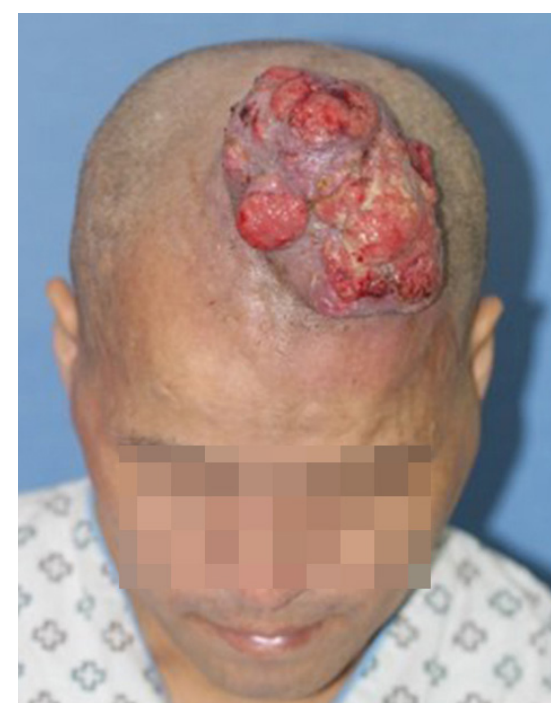

Fig. 3. Clinical photograph at 3 months after the first visit. The size of the mass has increased to $11 \times 11 \mathrm{~cm}$.

severe pain, and bleeding. Finally, the patient was surgically treated with wide local excision and radical lymph node dissection.

Under general anesthesia, wide local excision was performed, including excision of entire layers of the scalp with a 1-cm safety margin. In frozen sections, no tumor cells were observed at the resection margins, including the deep margin.

After resection, the size of the defect was measured as $13 \times 13$ $\mathrm{cm}$. We planned for a V-Y island flap for which the parietal branch of the left superficial temporal artery served as a pedicle. A triangular flap was designed on the posterior area of the defect. We performed a subcutaneous fat layer dissection from the side of the pedicle up to $3 \mathrm{~cm}$ above the ear. To elevate the flap, we performed a subgaleal dissection from the opposite side of the pedicle to $3 \mathrm{~cm}$ above the ear. The defect was reconstructed by advancement of the flap. The donor site defect was located on the posterolateral side of the vertex. To reduce the skin graft area and move it to the posterolateral side, an inferiorly based rotation flap using the occipital scalp was performed. The area remaining after the rotation flap was covered by split thickness skin graft. This was followed by bilateral radical neck lymph node dissection and lymph node biopsy.

The surgical biopsy revealed a large solid tumor characterized by ulcerative cutaneous lesions that occupied vast portions of the dermis with infiltrative growth. Moreover, there were welldefined, solid lobules of neoplastic cells composed of large, pale or clear cells with coarsely vacuolated cytoplasm and focal squamoid differentiation. Such neoplastic cells were well to moderately differentiated and had a lobular infiltrative pattern (Fig. 4). There was lymphatic invasion with neoplastic lym- 

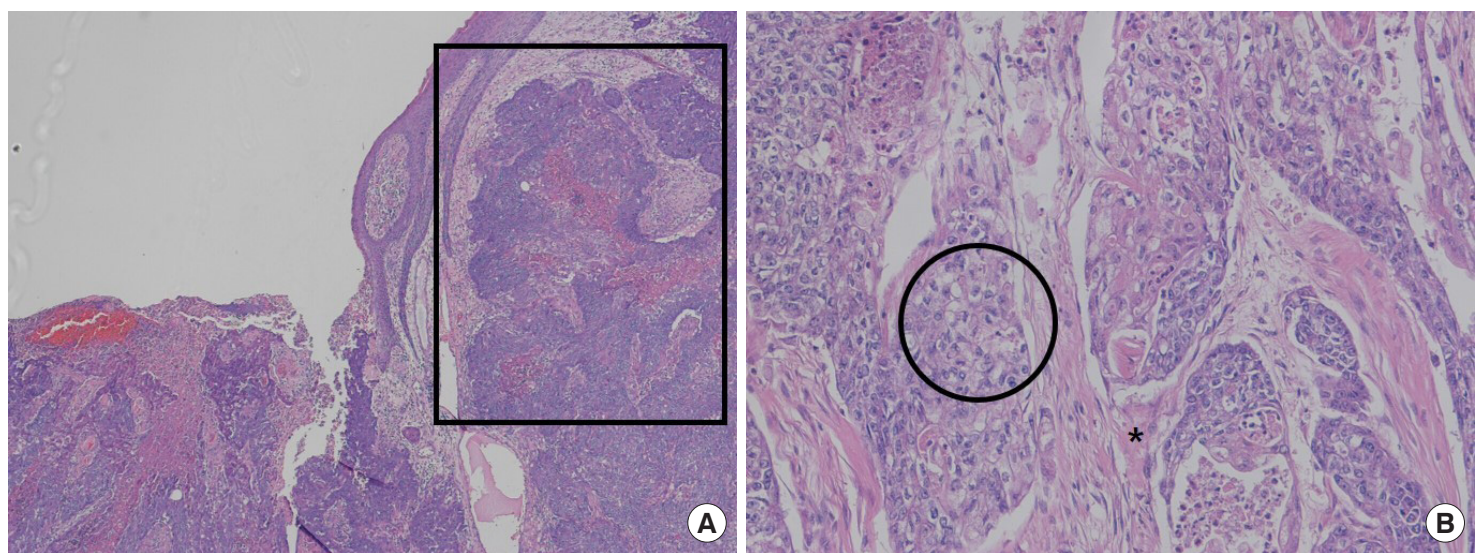

Fig. 4. Histopathologic findings. (A) An ulcerative cutaneous lesion is observed. Neoplastic cells are characterized by a lobular growth pattern (marked with a square) (H\&E, $\times 100)$. (B) Neoplastic cells contain well-defined solid lobules that are composed of large, pale or clear cells with coarsely vacuolated cytoplasm (marked with a circle) and focal squamoid differentiation (black asterisk) $(\mathrm{H} \& \mathrm{E}, \times 250)$.

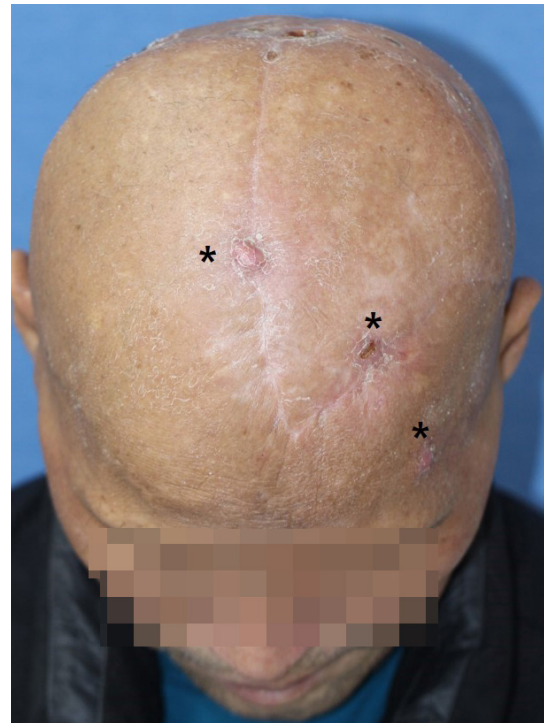

Fig. 5. Clinical photograph at 1 month after surgery. At 1 month postoperatively, recurrent lesions were observed (black asterisks).

phatic emboli, but there was no perineural invasion. The neoplastic cells also showed positive immunohistochemistry for epithelial membrane antigen. Moreover, a lymph node biopsy showed metastases to 13 of 84 lymph nodes. The patient was finally diagnosed with EOSC with lymph node metastases.

At a 1-month follow-up, the patient had three recurrent tumors, measuring $1 \times 1 \mathrm{~cm}$, on the surgical margin of the scalp (Fig. 5). Three months of radiation therapy was planned for the recurrent tumors. External beam radiotherapy was performed with doses of 200 Gy for the first 2 months and 250 Gy for the last month. Despite a decrease in the size of the masses, the patient did not achieve complete remission. Therefore, the patient underwent wide excision of the tumors with frozen section under local anesthesia. The defects were reconstructed by primary

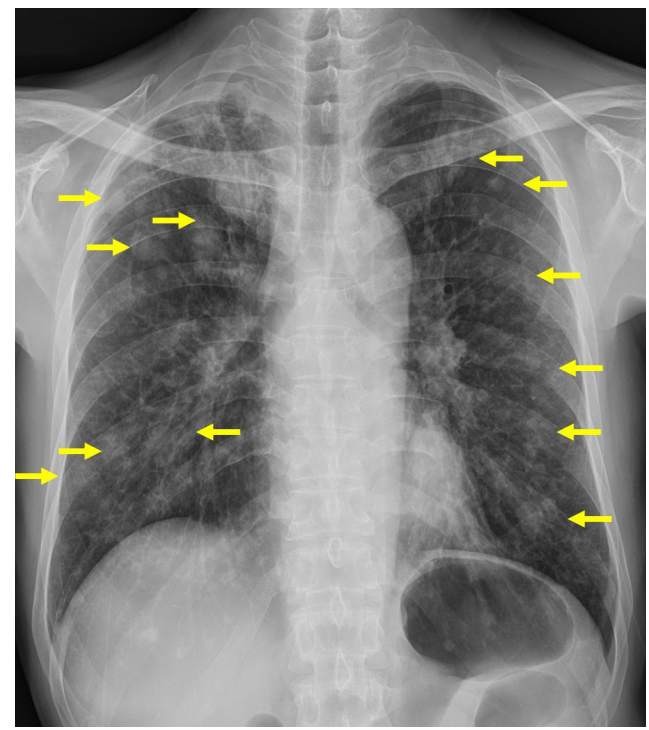

Fig. 6. Posterior-anterior chest radiography at 4 months after surgery for recurrent tumors. Multiple nodules (yellow arrows) are present in both lungs.

repair and rotation flap.

At 5 months, the patient complained of dyspnea even while walking on flat ground. Chest radiography revealed the presence of multiple nodules in both lungs, which were not previously present (Fig. 6). This was suggestive of lung metastases of SC. The patient was therefore planned to undergo chemotherapy but was again lost to follow-up.

\section{DISCUSSION}

In this case, the patient had a history of a patch-like scalp lesion that had not increased in size for 40 years. However, it suddenly began to grow and protrude 4 months before. Such a history 
suggested the possibility of malignant transformation of a benign lesion. Although rare, previous studies have reported cases of nevus sebaceous changes into SC [6,7]. The patient described the previous lesion as a yellow and hairless patch with an irregular surface accompanied by itching. Although it was not diagnosed histologically, it could have been a nevus sebaceous based on the clinical symptoms. In addition, Izumi et al. [7] reported that SCs arising from nevus sebaceous are characterized by pigmented patches, recent rapid increases in size, protruding patterns, and erosive surfaces. Since all these characteristics were observed in the patient in this study, it was presumed that SC occurred owing to malignant transformation of the nevus sebaceous.

SC can be classified as OSC and EOSC. It is known that 75\% of SCs are OSCs and 25\% of SCs are EOSCs [8]. Retrospective data have shown that $1.4 \%$ of EOSCs and $4.4 \%$ of OSCs are accompanied by metastases [4]; however, there is no difference in cancer-specific mortality between OSCs and EOSCs. The observed and relative survival rates of SC are $78.20 \%$ and $92.72 \%$, respectively, at 5 years and $61.72 \%$ and $86.98 \%$, respectively, at 10 years [2]. It has been reported that poorly differentiated tumors have a poorer prognosis compared to well-differentiated tumors [3]. Perineural involvement, lymphovascular invasion, noncontiguous multicentric growth pattern, higher $\mathrm{T}$ stage, and delayed diagnosis seem to be associated with poorer prognosis $[1-3,9]$.

Since only $1.4 \%$ of EOSCs are accompanied by metastases [4], there is a paucity of data about EOSCs accompanied by distant metastases. The common metastatic sites of EOSC are regional lymph nodes and visceral organs [5]. A variety of EOSC metastatic regions have been reported, including the lung, skin, liver, colon and brain [5,10-12].

It has been reported that the 8th American Joint Committee on Cancer (AJCC) TNM staging of cutaneous squamous cell carcinoma and other cutaneous carcinomas can be used for staging EOSCs. A study has shown that local recurrence and disease-specific survival outcomes can be predicted based on the $\mathrm{T}$ and $\mathrm{N}$ results of OSC [9]. However, there is no report on the relationship between TNM stage and the prognosis of patients with EOSC.

Owing to its rarity, the standard treatment of SC has not yet been established. It is known that the treatment of SC is excision of the primary tumor with wide local excision or Mohs micrographic surgery (MMS) [1-3]. According to a retrospective review, complete peripheral margin clearance could be expected with a mean safety margin of $0.54 \mathrm{~cm}$ [2]. Statistically, it is expected that $95 \%$ of tumors would be cleared with a $1.0 \mathrm{~cm}$ safety margin for EOSC [2]. However, there are no studies on deep margin resection. In the presence of lymph node metastasis, recommendations include resection and biopsy of the lymph node. The effectiveness of sentinel lymph node biopsy in $\mathrm{SC}$ without lymph node metastasis has yet to be proven [2]. For EOSC with distant metastases, radiation therapy, chemotherapy, and immunotherapy can be used. However, the effectiveness of these methods has not been established [3]. Chemotherapy and immunotherapy are not typical treatment methods in SC due to the small number of case reports $[2,3]$. The role of radiation therapy in recurrent tumors has been reported [3], but the number of reported cases is not large enough.

In this case, distant metastases were not initially observed. The tumor was larger than $2 \mathrm{~cm}$ in size with bilateral lymph node metastases, and the TNM stage was T2N2cM0. Since the tumor was on the scalp without involving the vital structure, it seemed possible to resect the primary tumor [13]. Therefore, according to the pre-existing treatment method, wide local excision of the primary tumor with a $1 \mathrm{~cm}$ safety margin and radical lymph node dissection were performed. However, local recurrence occurred within 1 month. Although the recurrent tumors were treated with radiation and surgical treatment, fatal distant metastases in both lungs occurred after 5 months.

The recurrence rate of EOSC is known to be $29 \%$, with an average period to recurrence of 19.4 months [3]. In this case, local recurrence occurred despite a clear surgical margin. Tumor recurrence in the surgical margin within less than 1 month suggests the possibility of residual tumor in the excision margin. Although the safety margin has been known to be $1 \mathrm{~cm}$, it seems that $1 \mathrm{~cm}$ was insufficient for such a large and aggressive tumor. In addition, SC is histologically characterized by noncontiguous multicentric growth patterns and pagetoid spread [14]. The noncontiguous multicentric growth pattern is a characteristic of OSCs. It is known to indicate a poor prognosis. Pagetoid spread is also a characteristic mainly associated with OSC, although it is rarely observed in EOSC. Some researchers have pointed out that observing pagetoid spread in frozen sections is difficult [15]. Thus, MMS is recommended over wide local excision for the treatment of SC [3]. In retrospective studies of OSC and EOSC, the recurrence rate after MMS is lower than that after wide local excision $[3,14]$. Based on these reports, MMS could have been an alternative option for this case. Radiation therapy is used as an adjuvant therapy in the treatment of EOSC. However, as radiation therapy is rarely performed to treat EOSC, the effectiveness of radiation therapy has not yet been proven. In a review of 1,349 cases, it was reported that only $5.3 \%$ of SC patients had been treated by surgery with adjuvant radiation therapy [16]. This combination therapy has been used for locally advanced tumors or those with positive 
resection margins. Chang et al. [17] suggested that postoperative radiation therapy is helpful in the treatment of EOSC. Giridhar et al. [18] reported the role of adjuvant radiation therapy in EOSC when it is characterized by positive margins, lymphovascular invasion, lymph node positivity, perineural invasion, bone infiltration, and anaplasia. As such, studies have highlighted the role of postoperative radiation therapy in the treatment of aggressive EOSC. Therefore, for the treatment of aggressive EOSC that is rapidly growing and has lymph node metastases, a combination therapy of surgery with adjuvant radiation therapy could be more beneficial than treatment with surgical therapy only.

In conclusion, we experienced a rare recurrent case of aggressive EOSC of the scalp with distant organ metastasis. We performed the pre-existing treatment for this case. However, to treat such aggressive tumors, it seems that other treatment options are needed. Since only a limited number of cases of aggressive EOSC have been reported, treatment modalities for aggressive EOSC have not been established. Therefore, further studies are warranted to determine the treatment options in this series.

\section{NOTES}

\section{Conflict of interest}

No potential conflict of interest relevant to this article was reported.

\section{Ethical approval}

The study was approved by the Institutional Review Board of Inje University Busan Paik Hospital (IRB No. 20-0175) and performed in accordance with the principles of the Declaration of Helsinki. Written informed consent was obtained.

\section{Patient consent}

The patient provided written informed consent for the publication and the use of his images.

\section{ORCID}

Byeong Soo Kwon

Eui Han Chung

Jin Woo Kim

\section{Author contribution}

Conceptualization: JWK. Project administration: JWK. Resources: JWK. Writing - original draft: BSK. Writing - review \& editing: BSK, EHC. Approval of final manuscript: all authors.

\section{REFERENCES}

1. Kyllo RL, Brady KL, Hurst EA. Sebaceous carcinoma: review of the literature. Dermatol Surg 2015;41:1-15.

2. Knackstedt T, Samie FH. Sebaceous carcinoma: a review of the scientific literature. Curr Treat Options Oncol 2017;18:47.

3. Owen JL, Kibbi N, Worley B, Kelm RC, Wang JV, Barker CA, et al. Sebaceous carcinoma: evidence-based clinical practice guidelines. Lancet Oncol 2019;20:e699-714.

4. Tryggvason G, Bayon R, Pagedar NA. Epidemiology of sebaceous carcinoma of the head and neck: implications for lymph node management. Head Neck 2012;34:1765-8.

5. Sawyer AR, McGoldrick RB, Mackey S, Powell B, Pohl M. Should extraocular sebaceous carcinoma be investigated using sentinel node biopsy? Dermatol Surg 2009;35:704-8.

6. Kim JY, Yang HJ, Lee JS, Lee HK, Kim JH. Sebaceoma arising from nevus sebaceous with early focal carcinomatous area. Arch Craniofac Surg 2016;17:93-5.

7. Izumi M, Tang X, Chiu CS, Nagai T, Matsubayashi J, Iwaya K, et al. Ten cases of sebaceous carcinoma arising in nevus sebaceus. J Dermatol 2008;35:704-11.

8. Tan O, Ergen D, Arslan R. Sebaceous carcinoma on the scalp. Dermatol Surg 2006;32:1290-3.

9. Sa HS, Rubin ML, Xu S, Ning J, Tetzlaff M, Sagiv O, et al. Prognostic factors for local recurrence, metastasis and survival for sebaceous carcinoma of the eyelid: observations in $100 \mathrm{pa}-$ tients. Br J Ophthalmol 2019;103:980-4.

10. Moreno C, Jacyk WK, Judd MJ, Requena L. Highly aggressive extraocular sebaceous carcinoma. Am J Dermatopathol 2001; 23:450-5.

11. Presman B, Tocco-Tussardi I, Jorgensen L, Birk-Sorensen L. Aggressive extraocular sebaceous carcinoma metastasizing to multiple visceral sites. J Case Rep Images Oncol 2015;1:23-7.

12. Lee JH, Shin HK, Jang TJ. A case of rapidly growing extraocular sebaceous carcinoma. Arch Craniofac Surg 2014;15:32-5.

13. Jang HU, Choi YW. Scalp reconstruction: a 10-year experience. Arch Craniofac Surg 2020;21:237-43.

14. Snow SN, Larson PO, Lucarelli MJ, Lemke BN, Madjar DD. Sebaceous carcinoma of the eyelids treated by mohs micrographic surgery: report of nine cases with review of the literature. Dermatol Surg 2002;28:623-31.

15. Folberg R, Whitaker DC, Tse DT, Nerad JA. Recurrent and residual sebaceous carcinoma after Mohs' excision of the primary lesion. Am J Ophthalmol 1987;103:817-23.

16. Dasgupta T, Wilson LD, Yu JB. A retrospective review of 1349 cases of sebaceous carcinoma. Cancer 2009;115:158-65.

17. Chang AY, Miller CJ, Elenitsas R, Newman JG, Sobanko JF. Management considerations in extraocular sebaceous carcino- 
ma. Dermatol Surg 2016;42Suppl 1:S57-65.

18. Giridhar P, Kashyap L, Mallick S, Dutt Upadhyay A, Rath GK. Impact of surgery and adjuvant treatment on the outcome of extraocular sebaceous carcinoma: a systematic review and individual patient's data analysis of 206 cases. Int J Dermatol 2020;59:494-505. 